\title{
Automatic and Effective Delineation of Coronary Arteries from CTA Data Using Two-Way Active Contour Model
}

\author{
Sammer ZAI ${ }^{\dagger \mathrm{a})}$, Member, Muhammad Ahsan ANSARI ${ }^{\dagger \mathrm{b})}$, and Young Shik MOON ${ }^{\dagger c)}$, Nonmembers
}

\begin{abstract}
SUMMARY Precise estimation of coronary arteries from computed tomography angiography (CTA) data is one of the challenging problems. This study focuses on automatic delineation of coronary arteries from 3D CTA data that may assess the clinicians in identifying the coronary pathologies. In this work, we present a technique that effectively segments the complete coronary arterial tree under the guidance of initial vesselness response without relying on heavily manual operations. The proposed method isolates the coronary arteries with accuracy by using localized statistical energy model in two directions provided with an automated seed which ensures an optimal segmentation of the coronaries. The detection of seed is carried out by analyzing the shape information of the coronary arteries in three successive cross-sections. To demonstrate the efficiency of the proposed algorithm, the obtained results are compared with the reference data provided by Rotterdam framework for lumen segmentation and the level-set active contour based method proposed by Lankton et al. Results reveal that the proposed method performs better in terms of leakages and accuracy in completeness of the coronary arterial tree.

key words: segmentation, computed tomography angiography, coronary artery, two-way active contour, vesselness response
\end{abstract}

\section{Introduction}

Cardiovascular diseases (CVDs) are one of the leading causes of disability and death in the developed countries [1]. An effective and timely detailed analysis is necessary to cure these life threatening diseases. To examine the coronary arteries without conventional coronary angiography (CCA) and intervention, the most widely used medical imaging technique is computed tomography angiography (CTA). CTA is considered as the state-of-the-art imaging modality which is proficient in obtaining $3 \mathrm{D}$ vascular information. However, due to the amount of data generated, it becomes inconvenient for radiologists to analyze axial images one by one. Here arises the need of robust segmentation technique that can help clinicians in accurate and fast diagnosis of coronary related abnormalities. The intra patient variation \& the complex structure of the arteries, makes the segmentation of vasculature a challenging task and hence it is an energetic area of research from early 90s. However, it continues to be an active research field because there is no single framework that fits all applications and requirements.

Manuscript received October 10, 2016.

Manuscript revised November 21, 2016.

Manuscript publicized December 29, 2016.

${ }^{\dagger}$ The authors are with Department of Computer Science and Engineering, Hanyang University, 1271 sa-3 dong, Ansan-si, Gyounggi-do, Korea.
a) E-mail: sammerzai09@ @anyang.ac.kr
b) E-mail: ansari05cs04@hanyang.ac.kr
c) E-mail: ysmoon@ hanyang.ac.kr
DOI: $10.1587 /$ transinf.2016EDP7419

The most widely used model for identifying and tracking of the vessels from complex $3 \mathrm{D}$ images is the active contour model because of its clear curve for the desired object [2]. The model evolves the contour under the impact of various forces. One of the popular representations is the Snake model presented by Kass et al. [3]. Their models was considered efficient in their early age, however it cannot represent the topological changes.

As a contrast to early works using image edges to formulate image-based energies, region based image features relying on global information derived from image regions, are more robust to noisy and inhomogeneous gradients [4]. The driving force based on the regional statistics makes the delineation process more powerful and also provides minimum dependency on the placement of the initial contour as compared to the edge based driving force. Various methods have been proposed in the literature that uses regional intensities for approximating the behavior of an image. In this context, famous algorithms have been reported by Chan and Vese [5] and Yezzi et al. [6], which were generally considered to be successful towards delineation of objects with weak and unclear gradient but they were inefficient to deal smoothed intensities exhibited by medical images. The model proposed by Chan and Vese is also known as piece wise constant model, which models the interior and exterior regions of the contour by globalized average intensity to obtain the precise segmentation. However, it produces inaccurate segmentation in the presence of intensity inhomogeneity that is usually contained by the medical image data [7]-[9].

Another technique has been proposed in the literature [4] which combined the intensity and shape information, and gave the improved segmentation result by preventing the contour leak into the neighboring regions. However, this method was time consuming for entropy computation and also sometimes it over-segments the vessels due to the adaptive scale selection scheme. Yang et al. [10] proposed incorporating active contour segmentation into a Bayesian probabilistic framework, where the image-driven energy is redefined by posterior probabilities. Localized active contours [11]-[13] have been introduced to improve the performance of the active contours in the presence of varying brightness across the image. Active contour based method is also used by Mohr and his co-workers [14] for segmenting the arteries lumen. They further utilized this segmentation for stenosis detection by performing the classification of vessel wall and calcium segmentation. Lankton and 
Tannenbaum [15] proposed the use of different metrics to measure the similar intensity distributions derived from image regions. In another approach, Lankton and his coworkers [16] have utilized localized active contour approach for detecting the coronary vessels by considering only the voxels representing the heart zone and ignoring very dark voxels representing air present in the lungs. However, their method requires a single-point initialization within the vessel by a user which may lead to erroneous segmentation and may also increase the processing time. Apart from this, their method often produces leakages while segmenting coronary arteries due to the leaning of contour into the nearby voxels exhibiting similar intensities. Requirement of the initial seed points from the user in order to initiate the region growing procedure has been presented in the literature [17]-[19]. However, these approaches were user dependent as they required seed point for every branch.

By considering the shortcomings of Lankton's approach [16], this study proposes to embed an automatic framework of fast seed detection with the level set based active contour model which is guided by the Hessian based vesselness in order to make the segmentation process automatic and leakage free. For detecting the coronary seed points without user interaction, we have adopted the idea of Dongjin et al. [20], where they have exploited the shape information of three main coronary arteries for the detection of seeds automatically. The detected seed point based on the geometrical analysis helps in generating the mask at a specified region which is provided as an input to the localized statistical deformable model. We run the localized curve in a way that it deforms itself in two directions to grab the potential side branches completely. The proposed method is an easy and automatic framework for efficiently extracting the coronaries from a 3D complex CTA data by first suppressing the non-vascular structures using the Hessian based filter prior to using the localized statistical energy model.

We now concisely explain the organization of the rest of this paper. In the subsequent section, we introduce some necessary background information. In Sect. 3, we explain about the proposed framework. Section 4 is dedicated to the evaluation of our approach where we also compare the proposed method with the previous method proposed by Lankton et al. [16], discuss the shortcomings of the method, and demonstrate the examples of precise and exact segmentations on challenging datasets. Finally, we make concluding remarks of this research in Sect. 5.

\section{Background Method}

\subsection{Localized Active Contour}

To deal with the intensity variation in medical images, Lankton et al. [15], has proposed a framework which permits the localization of region based segmentation energies with respect to spatial information. The energy is localized in a way that statistical models of inside and outside regions of the contour become adaptive to the information present in

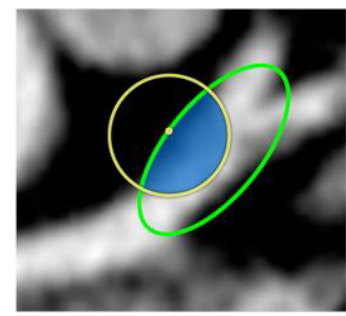

(a)

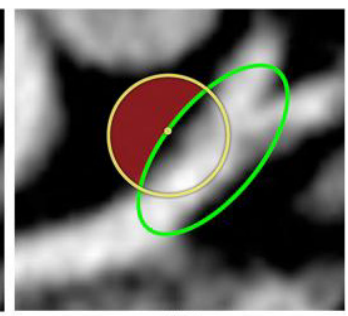

(b)
Fig. 1 Localized ball kernel to select local regions of contour (a) shaded region representing interior (b) shaded region representing exterior.

an image. In their method, a ball shaped characteristic function is used which is defined by Eq. (1), where $\mathrm{x}$ represents any point on the contour and y represents the point within a ball of radius $r$ centered at $\mathrm{x}$. The function computes the statistics of the region at each point of the curve. Figure 1 describes the behavior of the ball drawn at each point of the curve describing its local interior and exterior regions.

$$
\mathrm{B}(x, y)= \begin{cases}1, & \|x-y\|<r \\ 0, & \text { otherwise }\end{cases}
$$

By employing the concept of ball function the localized active contour minimizes energy functional defined by Eq. (2).

$$
\begin{gathered}
E(\phi)=\int_{\Omega x} \delta \phi(x) \int_{\Omega y} \mathrm{~B}(x, y) \cdot F(I, \phi, x, y) d y+ \\
\lambda \delta \phi(x)|\nabla \phi(x)| d x,
\end{gathered}
$$

where $\phi$ is a signed distance function which represents the contour such that $C=\{x \mid \phi(x)=0\}, \Omega$ represents the domain of a given intensity image I. The parameter $\lambda$ is a scalar weight on the arc-length and $F(I, \phi, x, y)$ indicates the internal energy functional. The interior region of the curve is defined by the Heaviside function, $\mathrm{H} \phi$ is equal to 1 when $\phi<0,0$ when $\phi>0$, and has a smooth transition through 0 . Similarly, the interface at the zero level set is represented by $\delta \phi$ (dirac delta function), the derivative of the Heaviside function $\mathrm{H} \phi$, which is 1 when $\phi=0$ and 0 far from the interface. In our study, we have formulated this internal energy as a well-known Chan Vese [5] energy defined by Eq. (3) which isolates the object from homogenous background on the basis of mean intensities of inner and outer regions of the contour. The parameters, $\mu_{1}$ and $\mu_{2}$ correspond to mean intensities of local inner and local outer regions, respectively. $I(y)$ indicates the local neighborhood point along the zero level set.

$$
F=\mathrm{H} \phi(y)\left(I(y)-\mu_{1}\right)^{2}+(1-\mathrm{H} \phi(y))\left(I(y)-\mu_{2}\right)^{2}
$$

Finally, the level set based evolution with respect to time $\mathrm{t}$ is defined by Eq. (4) by inserting the force in terms of piece wise constant model's energy.

$$
\begin{aligned}
& \frac{\partial \phi}{\partial t}(x)= \\
& \delta \phi(x) \int_{\Omega y} \mathrm{~B}(x, y) \delta \phi(y) \cdot\left(\left(I(y)-\mu_{1}\right)^{2}-\left(I(y)-\mu_{2}\right)^{2}\right) d y
\end{aligned}
$$




$$
+\lambda \operatorname{div}\left(\frac{\nabla \phi(x)}{|\nabla \phi(x)|}\right)|\nabla \phi(x)|
$$

\section{Proposed Approach}

To overcome the limitations of Lankton's approach [16], we propose an easy and automatic framework for extracting the coronaries efficiently from a 3D complex CTA data by first suppressing the non-vascular structures using the vessel enhancement filter prior to using the localized statistical energy model and an automatic seed detection procedure. The flow diagram of the overall process of the proposed approach is shown in Fig. 2.

Given a raw CTA volume, we have first scrutinized the CTA data by keeping only those voxels which represents the curvilinear structures using Hessian based vesselness filter. This refined output from the vesselness measure is made available simultaneously for the detection of coronary seed points as well to the localized deformable model. The detected seed helps in constructing the initial mask which will be updated after obtaining segmentation using initial mask to capture the side branches originating away from the main arteries. The resulting segmentation based on the curve evolution is added to the arterial tree at every step until the last slice is reached. The details of every step are described in subsequent sections.

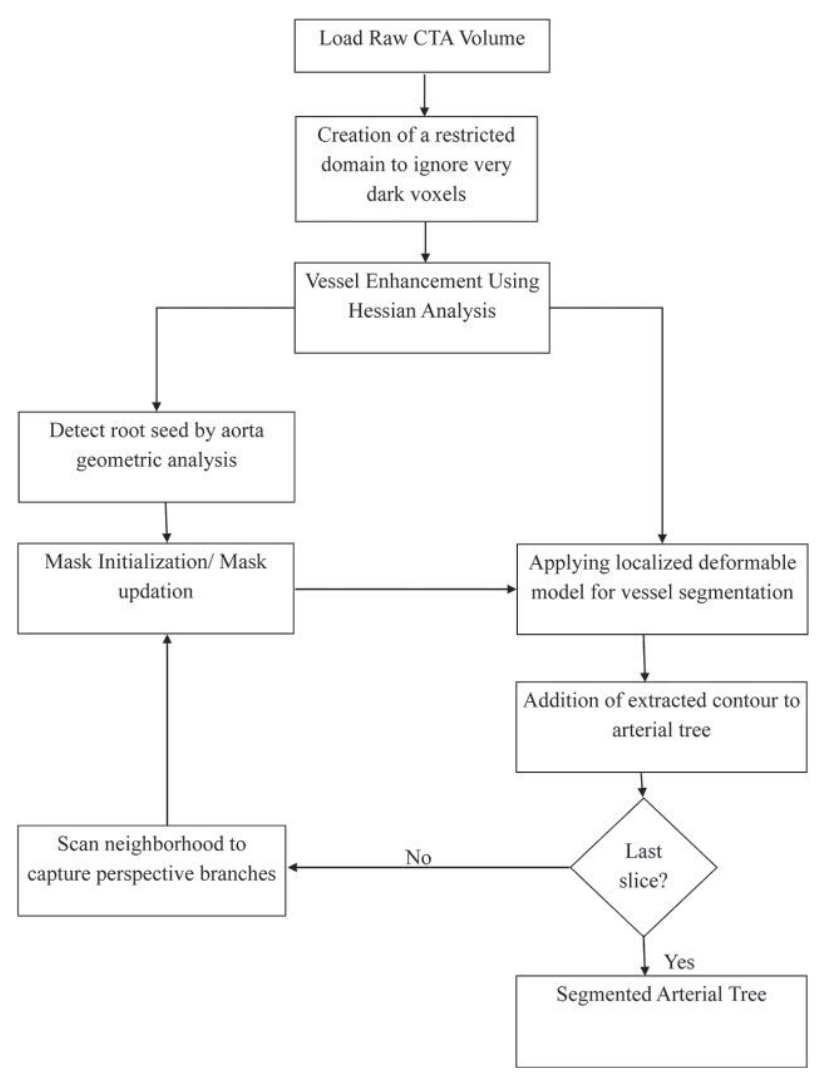

Fig. 2 Flow diagram of the proposed method

\subsection{Preliminary Step}

Since we are interested in coronary arteries only therefore prior to vessel enhancement and segmentation, a restricted domain is created in order to have only the voxels which represents the blood filled regions and ignoring the rest of voxels showing other organs. In order to have the blood filled regions we have thresholded the volume with respect to Hounsfield Units (HU). The threshold that we select here is $-600 \mathrm{HU}$ because this threshold is significantly below the ranges of blood and myocardial tissues in all CTA datasets [16].

\subsection{Extraction of Tubular Structures}

By considering the fact that the coronary arteries occupy only 2 to $2.5 \%$ of the complete CTA data [21], we propose to select only those voxels which shows tube-like structures. In our work, we have used the vesselness feature map developed by Frangi et al. [22] for differentiating the vessel and non-vessel components from volumetric dataset. The Hessian based vesselness is computed for the restricted environment which contains only the coronary artery components. This method uses the relationship among the eigenvalues of Hessian matrix for extracting tubular structures. Equation (5) is used to produce high values for the voxels that belongs to the tubular structures and vice versa. For the eigenvalues analysis of Hessian matrix, the eigenvalues $\lambda_{1}$, $\lambda_{2}$, and $\lambda_{3}$ are sorted as: $\left|\lambda_{1}\right|<\left|\lambda_{2}\right|<\left|\lambda_{3}\right|$ where $\left|\lambda_{1}\right| \approx 0$, $\left|\lambda_{1}\right| \ll\left|\lambda_{2}\right|$ and $\lambda_{2} \approx \lambda_{3}$ are the conditions for a perfect match with tube-like objects in a three dimensional dataset.

$$
\begin{aligned}
& f(x, \sigma)
\end{aligned}
$$

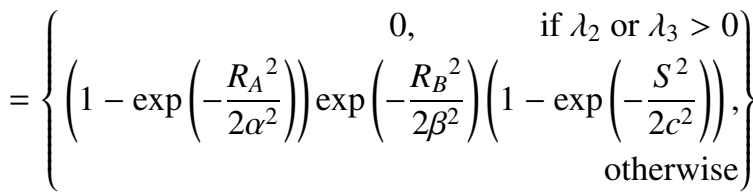

where,

$$
R_{A}=\frac{\left|\lambda_{2}\right|}{\left|\lambda_{3}\right|}, R_{B}=\frac{\left|\lambda_{1}\right|}{\sqrt{\left|\lambda_{2} \lambda_{3}\right|}} \quad \text { and } \mathrm{S}=\sqrt{\sum_{j} \lambda_{j}^{2}}
$$

The parameter $R_{A}$ differentiates between plate and tubelike components and the term $R_{B}$ checks for blob-like objects. Differentiation between vessel and non-vessel regions is handled by the parameter $\mathrm{S}$. The parameters $\alpha, \beta$ and $c$ controls the sensitivity of $R_{A}, R_{B}$ and $S$, respectively. To obtain a final estimate of vesselness, the vesselness measure defined by Eq. (5) is integrated at different scales $\sigma$ and the highest response at various scales is chosen as the best solution by using Eq. (6), where $\sigma_{\min }$ and $\sigma_{\max }$ define the minimum and maximum scales, respectively.

$$
F(x)=\max _{\sigma_{\min } \leq \sigma \leq \sigma_{\max }} f(x, \sigma)
$$




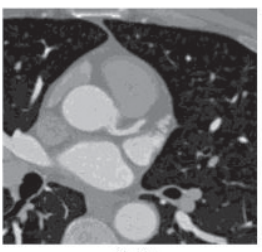

(a)

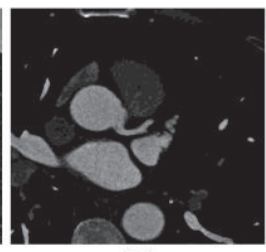

(b)

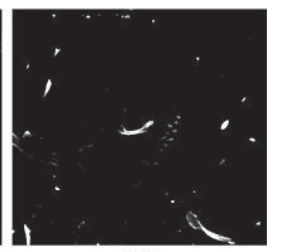

(c)
Fig.3 Pre-processing and vessel enhancement (a) Original axial slice showing the left coronary artery. (b) The resulting slice after performing thresholding by $-600 \mathrm{HU}$. (c) Frangi's vesselness.

The vessel enhancement measure $\mathrm{F}(\mathrm{x})$ given by Eq. (6) will be used to ensure that only coronary based voxels are selected during the curve evolution process. An example slice from a random CTA dataset containing the left coronary artery is shown in Fig. 3 (a) for which a restricted domain is created by thresholding the original CTA dataset with respect to Hounsfield Unit as shown in Fig. 3 (b). The Frangi vesselness is computed at each voxel as depicted by Fig. 3 (c). The vessel enhanced output is fed to the subsequent step of seed detection to detect the accurate coronary seeds.

\subsection{Automatic Seed Detection}

The accuracy of segmentation method depends upon the selection of seed points. As we have discussed in Sect. 1, manual definition of seed points may lead to an inaccurate segmentation, therefore an automatic method for seed selection is desired. In our study, we have exploited Dongjin's method [20] for seed detection where authors have proposed to use a local geometric feature in combination with hessian based filter to detect vessel regions. In their work, first an axial slice should be selected using the following equation:

$$
P=c_{r} * \mathrm{~N}
$$

where $\mathrm{N}$ corresponds to the total no. of slices in a given CTA volume and $c_{r}$ is a constant. After the selection of a CTA slice, region of interest is extracted by exploiting heart region detection technique [23] followed by sobel edge detection, opening operation, contour tracing and shape analysis procedure. The final coronary arterial seed points are selected using Eq. (8), which incorporates the local geometric feature defined by Eq. (9) into Hessian based vesselness. The parameter, $\mathrm{T}_{\mathrm{F}}$ represents the threshold for Hessianbased vesselness ' $\mathrm{F}(\mathrm{x})$ ' and $\mathrm{T}_{\mathrm{GF}}$ is the threshold for the local geometric feature ' $\mathrm{GF}(\mathrm{x})$ '.

$$
\text { vesselness }(x)=\left\{\begin{array}{cc}
1, & \text { if } F(x) \geq T_{F} \text { and } G F(x) \geq T_{G F} \\
0, & \text { otherwise }
\end{array},\right.
$$

where the local geometric feature, $\mathrm{GF}(\mathrm{x})$, is computed using Eq. (9).

$$
G F(x)=\prod_{j=1}^{10} \frac{k}{B_{\max }[j]-B_{\min }[j]+1},
$$

The local geometrical feature is computed by selecting three successive orthogonal cross-sections and casting of rays in 16 uniformly sampled directions across each plane. The radial gradient along each direction is recorded. Then according to the length, three largest and three smallest radiuses are discarded. Therefore, each cross-section has ten rays which are denoted by the variable $\mathrm{j}$. The minimum and maximum values $B_{\min }[j]$ and $B_{\max }[j]$, for each ray index, among the three planes are computed. The term ' $\mathrm{k}$ ' represents some constant. The final identified coronary pixels from 2D axial slice of contrast enhanced CTA will be used in subsequent stage as seed points for 3D surface construction of complete arterial tree.

The main strength of this method is fully automatic selection of initial seed points, which lead to complete automatic coronary segmentation in a robust manner. However, this method demands prior anatomical knowledge about coronary arteries in terms of shape information. The resulting output is used accordingly to guide the region growing process so that only coronary based voxels are selected during curve evolution process that will eventually lead to accurate segmentation.

\subsection{Mask Adaption}

In order to follow the arterial components more accurately and precisely we attempt to use a mask which will be reconstructed at the completion of every iteration. The initial mask is built on the basis of expected size of the lumen. By observing the anatomical behavior of the arterial tree, it is found that as the distance from the aorta increases, the arterial tree splits into further branches. However, there are certain cases, in which side branches originates away from the lumen and later connects the surface as axial slices are navigated as shown in Fig. 4. To cope with such situation, our algorithm adjusts the mask accordingly by persuing the voxels in the specific nearby region of the restricted area. The following algorithm contains the pseudo code for mask adaption.

\section{Algorithm}

1. Given an initial mask of the current slice, search for all possible candidates in the neighborhood of $15^{*} 15$.

2. For each possible candidate,

a. Perform edge detection using sobel operator.

b. Apply contour tracing to compute length and curvature. The curvature is defined and computed as follows:

$$
K=\sum_{i=1}^{L-1}|D[i+1]-D[i]|
$$

where $\mathrm{L}$ denotes the length of traced contour and $\mathrm{D}$ represents the direction of the move from one traced pixel to another. The variable $\mathrm{i}$ indicates the index of current traced pixel. 


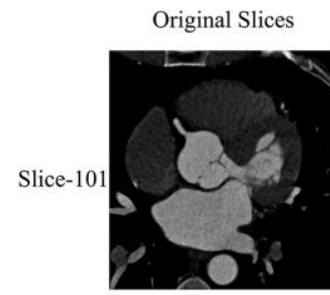

Lankton's Approach

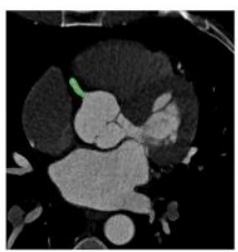

Proposed Approach
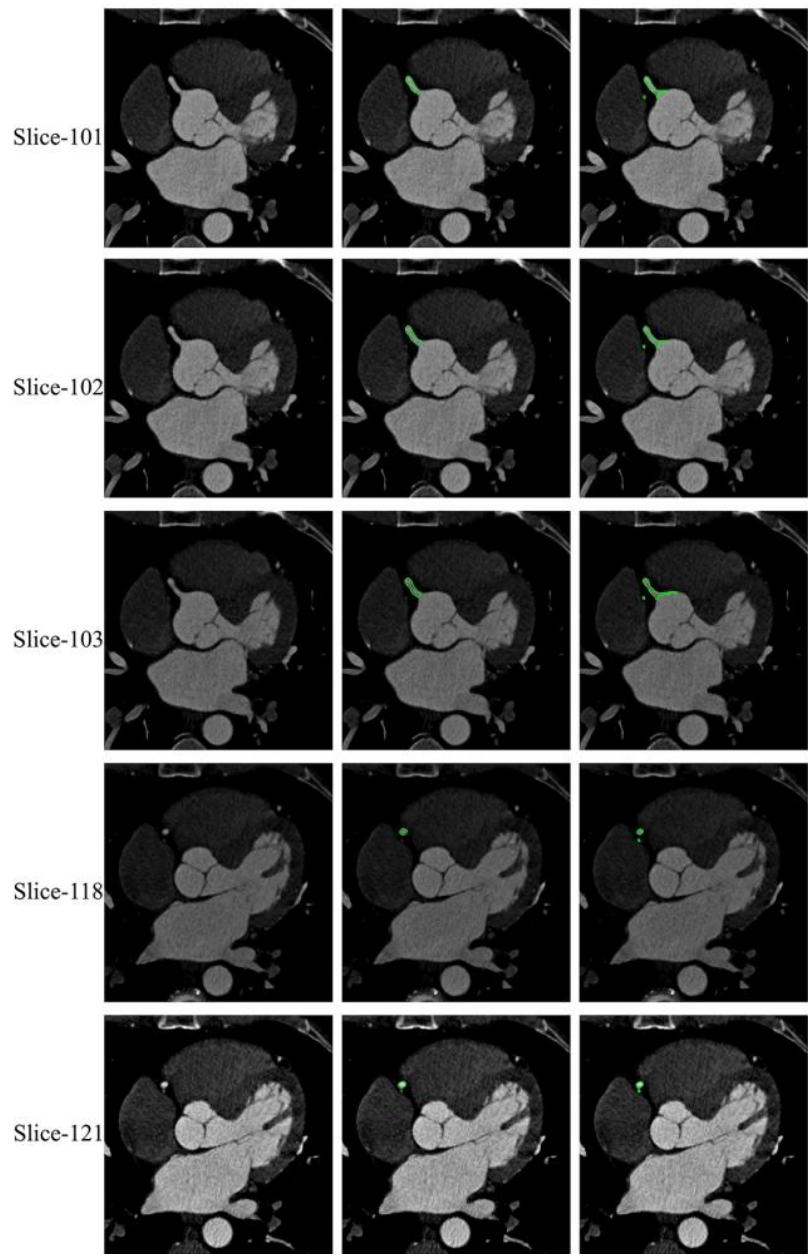

Fig. 4 Detection of side branches. First column: original axial slices showing component of right coronary artery along with side branch. Second column: failure of Lankton's approach in detecting the side branch. Third column: capturing of side branch using proposed mask adaption technique.

3. Discard all candidates having $\mathrm{L}>16$ and $\mathrm{K}<10$.

4. Threshold the remaining candidates having mean intensity greater than 160 .

The process of capturing the correct side branches for five different slices is shown in Fig. 4, where the first column shows the original CTA slice, second column represents the tracking of right coronary artery using Lankton's approach and third column indicates the side branches detected by the proposed method. It can be seen clearly, Lankton's approach is unable to detect the potential side branch which appears far from the main aorta. However, the proposed method detects the side branch accurately in all the slices as shown by the green circles in third column of Fig. 4.

The proposed mask adaption scheme guarantees the accuracy by detecting the potential side branches. It detects all the prospective branches that fulfill the geometric and intensity constraints as exhibited in Fig. 4. Further, morphological operations are performed to discard the components having no connectivity.

\subsection{Two-Way Segmentation}

Following the construction of initial mask, the localized active contour model based on the regional statistics is enforced for precise isolation and $3 \mathrm{D}$ reconstruction of arterial tree from CTA data. To resolve the inherent problem of intensity inhomogeneity in medical images we incorporate the localized regional intensity information in curve evolution as described by Eq. (4) for tracking the full coronary arterial tree.

The accuracy of the proposed algorithm depends upon the detected seed points and the reference slice used for seed detection. This is notable that the arterial information exists before and after the reference slice. Therefore to retrieve the complete arterial tree accurately the proposed algorithm is executed in both directions such that from reference slice to the ending slice and from reference slice to the first slice.

The localized Chan-Vese model was also used by Lankton et al. [16], but in their approach segmentation often produces leakages due to the minor distinction in intensities that isolate the vessels from nearby structures. In our method, we have overcome these leakages by guiding the contour with the vesselness feature map as we have described in Sect. 3.2. Since the enhanced vesselness measure contains only refined data of coarse tubular geometry, hence it can be used to direct the active contour model for preventing leakages in other blood filled regions. The use of the proposed framework produces accurate segmentation of the main vessels $(\mathrm{LCA}=$ Left Coronary Artery, LCX $=$ Left Circumflex and RCA = Right Coronary Artery) entirely without producing leakages and requiring aid from the user.

\section{Experimental Results}

This section explains the effectiveness of the proposed framework to a challenging task that is accurate and exact segmentation of coronary arteries. The efficiency of the framework is shown with the help of statistical data as well as with visual analysis after verifying it from the radiologist. The algorithm is tested on ten real clinical CTA datasets as well as we have used 18 real clinical datasets of symptomatic patients with the consensus of expert opinions provided by Rotterdam framework which are available publically. These datasets include the image data of patients who presented either stable or unstable angina syndromes, and who underwent both CTA and CCA examinations. Datasets were retrospectively acquired in three university hospitals, and evaluated anonymously. Thus, no IRB approval was required, according to the ethics committee guidelines of the involved medical centers [24].

The CTA datasets have been acquired from CT scanners of different vendors. The specifications of these scanners are presented in Table 1. The average voxel size of the datasets is $0.37 * 0.37 * 0.36 \mathrm{~mm}^{3}$. All images are reconstructed at $512 * 512$ size with varying number of slices. The datasets belong to the patients aged between 41 and 80 years 
Table 1 Specifications of CT scanners used for acquiring datasets.

\begin{tabular}{|l|l|l|l|}
\hline Vendor & $\begin{array}{l}\text { Siemens Medical } \\
\text { Solutions, } \\
\text { Forchheim, Germany }\end{array}$ & $\begin{array}{l}\text { Toshiba } \\
\text { Medical } \\
\text { Systems, } \\
\text { Tokyo, Japan }\end{array}$ & $\begin{array}{l}\text { Philips Medical } \\
\text { Systems, Best, } \\
\text { the Netherlands }\end{array}$ \\
\hline Institution & $\begin{array}{l}\text { Erasmus MC, } \\
\text { University Medical } \\
\text { Center Rotterdam } \\
\text { (NL) }\end{array}$ & $\begin{array}{l}\text { LUMC, Leiden } \\
\text { University } \\
\text { Medical Center } \\
\text { (NL) }\end{array}$ & $\begin{array}{l}\text { UMCU, } \\
\text { University } \\
\text { Medical Center } \\
\text { Utrecht (NL) }\end{array}$ \\
\hline $\begin{array}{l}\text { CT Scanner } \\
\text { Slice per } \\
\text { rotation }\end{array}$ & $32 * 2$ & Aquilion 320 & Brillance 64 \\
\hline $\begin{array}{l}\text { Number of } \\
\text { datasets }\end{array}$ & 6 & $320 * 1$ & $64 * 1$ \\
\hline
\end{tabular}

Table 2 Patient's information.

\begin{tabular}{|l|l|l|l|l|}
\hline & All & EMC & UMCU & LUMC \\
\hline Scan date & & & & \\
Min. & $06 / 2005$ & $08 / 2006$ & $06 / 2005$ & $06 / 2008$ \\
Max. & $06 / 2011$ & $12 / 2008$ & $06 / 2006$ & $06 / 2011$ \\
\hline Age & & & & \\
Mean \pm & $58.76 \pm$ & $58.8 \pm$ & $57.31 \pm$ & $60.17 \pm$ \\
std & 8.71 & 11.05 & 7.25 & 7.05 \\
{$[$ min,max $]$} & {$[41,80]$} & {$[43,80]$} & {$[41,69]$} & {$[52,74]$} \\
\hline Gender & & & & \\
Males (\%) & $32(67 \%)$ & $12(75 \%)$ & $11(69 \%)$ & $10(63 \%)$ \\
\hline CV risk & & & & \\
factors & $6(13 \%)$ & $0(0 \%)$ & $3(19 \%)$ & $3(19 \%)$ \\
Obesity & $20(42 \%)$ & $3(18 \%)$ & $8(50 \%)$ & $9(56 \%)$ \\
Smoking & $21(44 \%)$ & $7(44 \%)$ & $6(38 \%)$ & $8(50 \%)$ \\
Hypertension & $5(10 \%)$ & $0(0 \%)$ & $1(6 \%)$ & $4(25 \%)$ \\
Diabetes & $23(48 \%)$ & $956 \%)$ & $6(38 \%)$ & $8(50 \%)$ \\
Fam. Hist. & & & & \\
\hline
\end{tabular}

old, enrolled in three university hospitals between June 2005 and June 2011. The characteristics of patients are listed in Table 2. Additional information can be found on the website (hhtp://coronary.bigr.nl/stenosis/about.php).

Level set approach is famous to represent a contour because of its simple implementation and its behavior to automatically adopt the topological changes. However, these methods are very slow in computation. Therefore, to implement our algorithm we have used the sparse field technique developed by Whitaker [25] to implement level set active contours efficiently. In our study, the range of scales $\sigma$ for finding the vesselness measure is 2 to 5 . Also, for computing Eq. (8), we have used $\mathrm{T}_{\mathrm{F}}=0.009$ and $\mathrm{T}_{\mathrm{GF}}=18$. For our experiments, the value of constant $\mathrm{k}$ in Eq. (9) is set to 2 .

As we have discussed in Sect. 3.4, the loss of arterial information occurs depending upon the location of the reference slice. If the reference slice is exactly axial cross section where respective coronary artery comes out of aorta, complete tree can be segmented efficiently. However, due to the condition that same reference slice to be used for both seed points (left and right coronary artery), usually middle of CTA volume is chosen as reference slice by putting value of $c_{r}$ in the range of $[0.4,0.6]$. This middle slice based seed point detection is allocated with the facility to perform segmentation in the backward direction by navigating through all the slices in a reverse order. The resulting segmentation of the coronary arteries is the logical mixture of two way segmentations (forward and backward), therefore contains
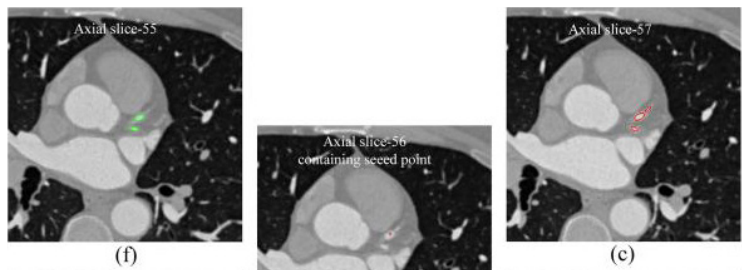

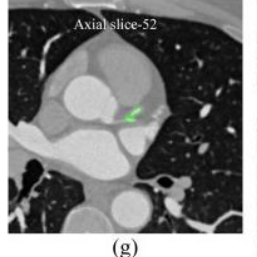

(g)
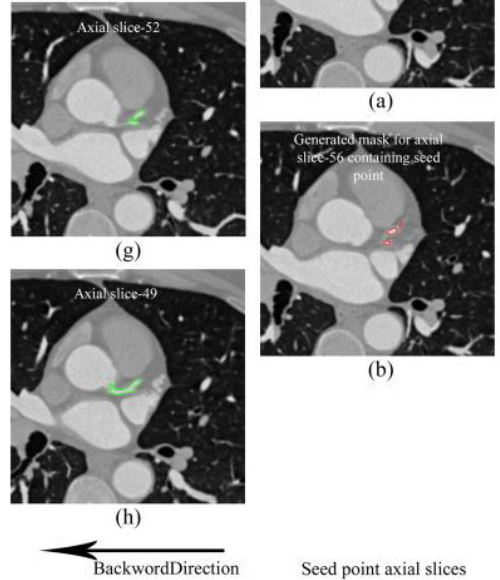

(a)
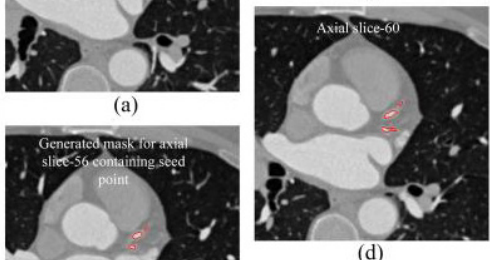

(d)

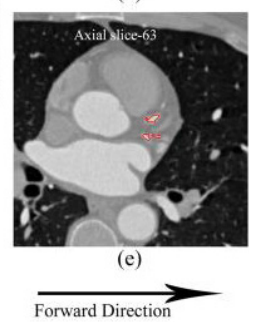

Fig. 5 Illustration of two-way segmentation where green contour represents backward segmentation and red contour represent forward segmentation. (a) Axial slice-56 segmented. (b) Axial slice-56 containing seed. (c) Axial slice-57 segmented. (d) Axial slice-60 segmented. (e) Segmentation for axial slice-63. (f) Axial slice-55 segmented. (g) Segmentation for axial slice-52. (h) Segmentation for axial slice-49.

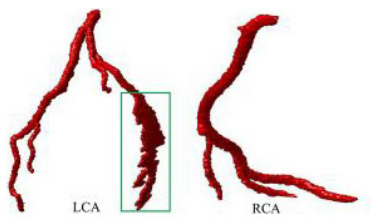

(a)

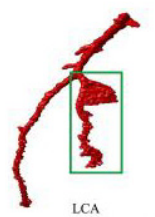

LCA

(c)

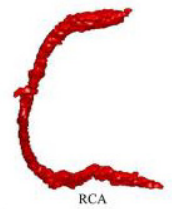

)

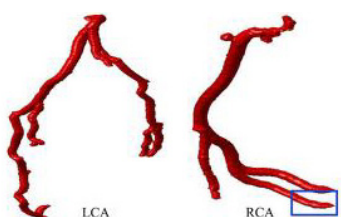

(b)

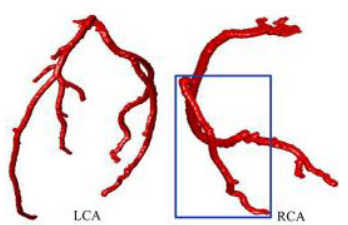

(d)
Fig. 6 Comparison of segmentation on two different datasets. (a) Segmentation of CTA Volume-1 using Lankton's method. (b) Segmentation of CTA Volume-1 using proposed method. (c) Segmentation of CTA Volume2 using Lankton's method. (d) Segmentation of CTA Volume-2 using proposed method.

the complete knowledge of the coronary arteries. Figure 5 shows the segmentation in forward direction with red contours and backward direction with green contours.

Superior results of the proposed algorithm can be confirmed by looking at Fig. 6 which shows the obtained segmentation results for left and right coronary arteries for two randomly selected CTA datasets. Figure 6(a) and (c), represents the segmentation obtained through the Lankton's approach. Whereas, Fig.6(b) and (d), shows respective segmentation obtained using proposed method. 
Table 3 TP, TN, FP and JI values for the segmentations of both CTAVolumes shown in Fig. 6.

\begin{tabular}{|c|c|c|c|c|c|c|c|c|c|c|c|c|c|c|c|c|}
\hline & \multicolumn{8}{|c|}{ CTA-Volume-1 } & \multicolumn{8}{|c|}{ CTA-Volume-2 } \\
\hline & \multicolumn{4}{|c|}{ LCA } & \multicolumn{4}{|c|}{ RCA } & \multicolumn{4}{|c|}{ LCA } & \multicolumn{4}{|c|}{ RCA } \\
\hline & TP & TN & FP & J & TP & TS & NFP & ال & TP & TN & FP & J & TP & TN & FP & J \\
\hline Lankton & 80 & 20 & 35 & 0.69 & 85 & 15 & 6 & 0.6 & 55 & 45 & 25 & 0.48 & 60 & 40 & 12 & 0.53 \\
\hline Proposed & 87 & 13 & 20 & 0.72 & 87 & 13 & 12 & 0.7 & 85 & 15 & 16 & 0.75 & 88 & 12 & 21 & 0.74 \\
\hline
\end{tabular}

As can be observed in Fig. 6 (a) and (c), Lankton's method produces leakages marked by green boxes, due to the slight differences in intensities. Whereas, the guidance of the localized energy model through the vessel enhancement procedure of the proposed method prevents the contour to leak into the adjacent regions during curve propagation, thereby producing segmentation without leakages as shown in Fig. 6(b) and (d). It is also observed that the proposed technique confirms that both left and right coronaries are well tracked from the aorta to the distal points as compared to the Lankton's approach. The areas marked by blue boxes in Fig. 6(b) and (d) exhibits the tracking of arteries to their distal ends which are missing in Lankton's approach as shown in Fig. 6 (a) and (c). One more benefit of the proposed algorithm is that the complete coronary arterial tree can be extracted at once including the major and the side branches without human interactions as compared to Lankton's method which requires a single point initialization for every artery separately. Table 3 gives the quantitative comparison of the proposed method and the Lankton's approach for the segmentation results of both CTA volumes shown in Fig. 6 in terms of True Positive (TP), True Negative (TN), False Positive (FP) and Jaccard Index (JI).

All the obtained segmentations are also compared with the reference segmentations for eighteen datasets provided by the Rotterdam framework. The reference data consist of the boundary points for each segment of the artery. To compare with the reference contours, the obtained segmented arterial trees are first converted into their equivalent mesh and then medial axis of these segmented results are generated using pre-defined fast marching implementation of sub-voxel skeletonization algorithm [26]. Resulting centerlines are portioned into different segments according to 17segment model of American Heart Association (AHA) and the boundary points are obtained. Finally, the obtained contours are compared with that of the reference contours in terms of TP, TN and FP. Hence, achieved segmentations are evaluated and the performance of our method is compared with that of the three reference contours produced by three independent observers manually. The quantitative results for the segmentation accuracy are provided in Table 4, where the average results of our method along with standard deviation for all datasets with respect to all three observers
Table 4 Average segmentation accuracy.

\begin{tabular}{lllll}
\hline & \multicolumn{1}{c}{ TP } & \multicolumn{1}{c}{ TN } & FP & JI \\
\hline Observer-1 & 87.46 & 12.54 & 20.92 & 0.76 \\
Observer-2 & 86.76 & 13.24 & 21.56 & 0.72 \\
Observer-3 & 85.43 & 14.57 & 32.32 & 0.66 \\
Average \pm std & $86.5 \pm 2.22$ & $13.45 \pm 2.22$ & $24.93 \pm 9.07$ & $0.71 \pm 0.04$ \\
\hline
\end{tabular}

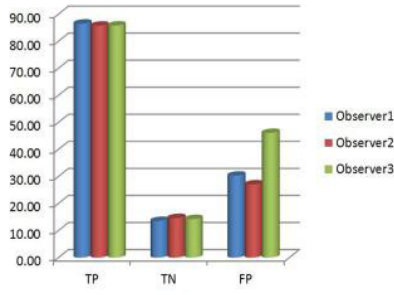

(a)

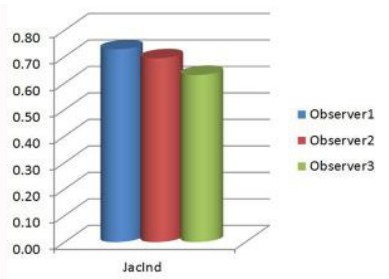

(b)
Fig. 7 Graphical results with respect to observer 1,2 and 3. (a) Graph showing TP, TN and FP. (b) Graph showing JI.

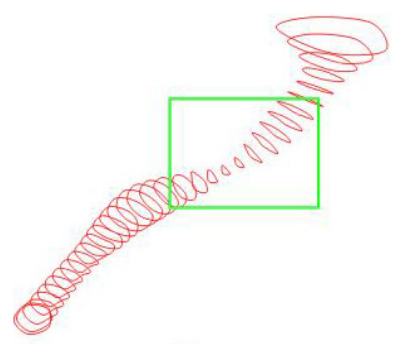

(a)

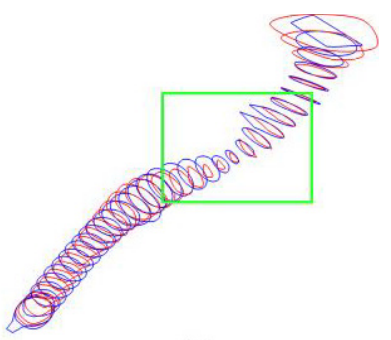

(b)
Fig. 8 Example of obtained and the reference contour.

are shown. The measure, Jaccard index (JI) is used for measuring the similarity of our method with the observers. The graphical results showing TP, TN, FP and JI with respect to observer 1, 2 and 3 are shown in Fig. 7.

By looking at the results given in Table 4, it can be observed that the proposed method achieves almost $71 \%$ similarity on average with that of observer's segmentation. However, it should be noted that the current goal of our research is the complete lumen segmentation without quantifying plaques. But the reference segmentations have been provided by excluding the plaque affected regions as a result the lumen becomes narrow or occluded for those regions containing plaque as shown by the green box in Fig. 8 (a). For more clear understanding we have shown an example of our segmentation in Fig. 8 (b) by overlapping the contours of obtained segmentation and the reference segmentation, where blue contours represent our segmentation and the red contours represent the reference segmentation. As can be seen clearly in the area enclosed by the green box, the reference contours are narrow due to the existence of plaque. This is the primary reason of high false positives when comparing our segmentation with that of the reference segmentation. Moreover, there is not a perfect agreement among all three observers due to the manual segmentation of coronary arteries which depends on their expertise. Therefore, the results are slightly different for all three observers.

Finally, the average processing time of the proposed method is $15 \pm 5$ minutes on $2.14 \mathrm{GHz}$ processor with $4 \mathrm{~GB}$ 
RAM. Implementation of the proposed method has been done on MATLAB R2015a installed on windows 7, 64-bit Operating System.

\section{Conclusion}

A simple yet effective method for accurate segmentation of coronary arteries has been presented by incorporating the vesselness information to help the contour to identify the object of interest accurately. The proposed guidance to the localized energy model through the vessel enhancement procedure also prevents the contour to leak into the adjacent regions during curve propagation thereby, producing segmentation without leakages. Moreover, the shape features of coronary vessels have also been incorporated for an automatic seed detection which has made this framework robust and independent of the user. The two-way segmentation has been applied to avoid loss of coronary information due to the seed point location whereas auto adjustment feature of the contour grabs new emerging branches. The proposed method has been validated on publically available datasets of the Rotterdam Coronary Artery Evaluation Framework. Additionally, the obtained results are compared with that of Lankton's method to demonstrate the superior performance of the proposed algorithm. Both qualitative and quantitative results exhibit the efficiency of the proposed method. Future work includes the improvement of statistical results and the refinement of the segmentation algorithm with the aim of detecting soft plaques present in the coronary arteries.

\section{References}

[1] T. Jerman, F. Pernus, B. Likar, and Z. Spiclin, "Beyond Frangi: an improved multiscale vesselness filter," Proc. SPIE Medical Imaging, vol.9413, March 2015.

[2] T. Liu, H. Xu, W. Jin, Z. Liu, Y. Zhao, and W. Tian, "Medical image segmentation based on a hybrid region-based active contour model," Computational and mathematical methods in medicine, vol.2014, 2014.

[3] M. Kass, A. Witkin, and D. Terzopoulos, "Snakes: Active contour models," International journal of computer vision, vol.1, no.4, pp.321-331, 1988.

[4] Y. Wang and H. Jiang, "A nonparametric shape prior constrained active contour model for segmentation of coronaries in CTA images," Computational and mathematical methods in medicine, vol.2014, no.1, April 2014.

[5] T.F. Chan and L.A. Vese, "Active contours without edges," IEEE Trans. Image Process., vol.10, no.2, pp.266-277, Jan. 2001.

[6] A. Yezzi, A. Tsai, and A. Willsky, "A fully global approach to image segmentation via coupled curve evolution equations," Journal of Visual Communication and Image Representation, vol.13, no.1-2, pp.195-216, March 2002.

[7] L. Wang, C. Li, Q. Sun, D. Xia, and C.-Y. Kao, "Active contours driven by local and global intensity fitting energy with application to brain MR image segmentation," Computerized Medical Imaging and Graphics, vol.33, no.7, pp.520-531, June 2009.

[8] J. An, M. Rousson, and C. Xu, " $\gamma$-convergence approximation to piecewise smooth medical image segmentation," Medical Image Computing and Computer-Assisted Intervention-MICCAI, vol.10, no.2, pp.495-502, 2007.

[9] J. Piovano, M. Rousson, and T. Papadopoulo, "Efficient segmentation of piecewise smooth images," in Scale Space and Variational
Methods in Computer Vision, pp.709-720, May 2007.

[10] Y. Yang, A. Tannenbaum, D. Giddens, and A. Stillman, "Automatic segmentation of coronary arteries using bayesian driven implicit surfaces," Proc. 4th IEEE International Symposium on Biomedical Imaging: From Nano to Macro, ISBI 2007, pp.189-192, May 2007.

[11] M.W.K. Law and A.C.S. Chung, "Weighted local variance-based edge detection and its application to vascular segmentation in magnetic resonance angiography," IEEE Trans. Med. Imag., vol.26, no.9, pp.1224-1241, Oct. 2007

[12] C. Li, C.-Y. Kao, J.C. Gore, and Z. Ding, "Implicit active contours driven by local binary fitting energy," Proc. IEEE Computer Vision and Pattern Recognition, pp.1-7, July 2007.

[13] C. Li, C.-Y. Kao, J.C. Gore, and Z. Ding, "Minimization of regionscalable fitting energy for image segmentation," IEEE Trans. Image Process., vol.17, no.10, pp.1940-1949, Nov. 2008.

[14] B. Mohr, S. Masood, and C. Plakas, "Accurate lumen segmentation and stenosis detection and quantification in coronary CTA," Proc. Int. Conf. 3D Cardiovascular Imaging: A MICCAI Segmentation Challenge Workshop, 2012.

[15] S. Lankton and A. Tannenbaum, "Localizing region-based active contours," IEEE Trans. Image Process., vol.17, no.11, pp.2029-2039, Dec. 2008

[16] S. Lankton, A. Stillman, P. Raggi, and A.R. Tannenbaum, "Soft plaque detection and automatic vessel segmentation," Proc. Probabilistic Moels for Medical Image Analysis: A MICCAI Workshop, Sept. 2009.

[17] H. Scherl, J. Hornegger, M. Prümmer, and M. Lell, "Semi-automatic level-set based segmentation and stenosis quantification of the internal carotid artery in 3D CTA data sets," Medical image analysis, vol.11, no.1, pp.21-34, Feb. 2007.

[18] A. Khedmati, A. Nikravanshalmani, and A. Salajegheh, "Semi-automatic detection of coronary artery stenosis in 3D CTA," IET Image Processing, vol.10, no.10, pp.724-732, Oct. 2016.

[19] I. Oksuz, D. Unay, and K. Kadıpaşaoğlu, "A hybrid method for coronary artery stenoses detection and quantification in CTA images," Proc. Int. Conf MICCAI Workshop, 3D Cardiovascular Imaging: A MICCAI Segmentation, Jan. 2012.

[20] D. Han, N.-T. Doan, H. Shim, B. Jeon, H. Lee, Y. Hong, and H.-J. Chang, "A fast seed detection using local geometrical feature for automatic tracking of coronary arteries in CTA," Computer methods and programs in biomedicine, vol.117, no.2, pp.179-188, Nov. 2014.

[21] L. Linsen, H. Hagen, and B. Hamann, ed., Bernd, Visualization in medicine and life sciences, Springer, 2008.

[22] A.F. Frangi, W.J. Niessen, K.L. Vincken, and M.A. Viergever, "Multiscale vessel enhancement filtering," Proc. Medical Image Computing and Computer-Assisted Interventation-MICCAI'98, ed: Springer, pp.130-137, 1998.

[23] Y. Wang, Blood vessel segmentation and shape analysis for quantification of coronary artery stenosis in CT angiography, Ph.D., School of Engineering and Mathematical Sciences, City University London, 2011.

[24] H.A. Kirişli, M. Schaap, C.T. Metz, A.S. Dharampal, W.B. Meijboom, S.L. Papadopoulou, A. Dedic, K. Nieman, M.A. de Graaf, M.F.L. Meijs, M.J. Cramer, A. Broersen, S. Cetin, A. Eslami, L. Flórez-Valencia, K.L. Lor, B. Matuszewski, I. Melki, B. Mohr, I. Öksüz, R. Shahzad, C. Wang, P.H. Kitslaar, G. Unal, A. Katouzian, M. Orkisz, C.M. Chen, F. Precioso, L. Najman, S. Masood, D. Ünay, L. van Vliet, R. Moreno, R. Goldenberg, E. Vuçini, G.P. Krestin, W.J. Niessen, and T. van Walsum, "Standardized evaluation framework for evaluating coronary artery stenosis detection, stenosis quantification and lumen segmentation algorithms in computed tomography angiography," Medical image analysis, vol.17, no.8, pp.859-876, June 2013.

[25] R.T. Whitaker, "A level-set approach to 3D reconstruction from range data," International journal of computer vision, vol.29, no.3, pp.203-231, Sept. 1998. 
[26] R. Van Uitert and I. Bitter, "Subvoxel precise skeletons of volumetric data based on fast marching methods," Medical physics, vol.34, no.2, pp.627-638, March 2007.

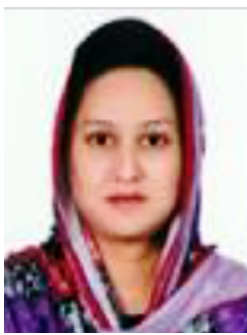

Sammer Zai received the B.E. and M.E. degrees in Computer Science Engineering and Communication Systems and Networks Engineering from Mehran University of Engineering and Technology in 2008 and 2012, respectively. She is currently working towards her PhD degree at computer vision and pattern recognition laboratory, department of Computer Science and Engineering, Hanyang University, Republic of Korea.

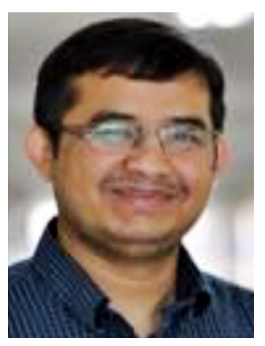

Muhammad Ahsan Ansari received the B.E. and M.E. degrees in Computer Science Engineering and Communication Systems and Networks Engineering from Mehran University of Engineering and Technology in 2008 and 2012, respectively. He is currently working towards his $\mathrm{PhD}$ degree at computer vision and pattern recognition laboratory, department of Computer Science and Engineering, Hanyang University, Republic of Korea.

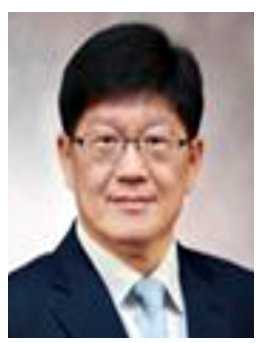

Young Shik Moon received the B.S. and M.S. degrees in Electronics Engineering from Seoul National University and Korea Advanced Institute of Science and Technology, Korea, in 1982, respectively, and PhD. Degree in Electrical and Computer Engineering from the University of California at Irvine, CA, in 1990. From 1982 to 1985 , he had been a researcher at the Electronics and Telecommunication Research Institute, Daejon, Korea. In 1992, he joined the department of Computer Science and Engineering at Hanyang University, Korea, as an Assistant Professor, and is currently a Professor. Dr. Moon served as General Chair of 2014 IEEE International Symposium on Consumer Electronics, and worked as the President of the Institute of Electronics and Information Engineer, Korea. 